\title{
Body Composition and Anthropometry in Bodybuilders: Regional Changes due to Nandrolone Decanoate Administration
}

\author{
F. Hartgens ${ }^{1}$, W. D. Van Marken Lichtenbelt², S. Ebbing ${ }^{1}$, N. Vollaard², G. Rietjens ${ }^{1,3}$, H. Kuipers ${ }^{3}$ \\ ${ }^{1}$ Netherlands Centre for Doping Affairs, Capelle aan den IJssel, The Netherlands \\ 2 Department of Human Biology, Maastricht University, The Netherlands \\ ${ }^{3}$ Department of Movement Sciences, Maastricht University, The Netherlands
}

\section{Erratum}

We do apologize for an incorrect specification published in issue $3 / 2001$ on page 236 . In the article of F. Hartgens et al. in section materials and methods the manufacturer of nandrolone decanoate reads correctly Organon B. V., Oss, The Netherlands, not Novartis. 